\title{
Anopheline Mosquitoes and the Malaria Scourge
}

\author{
Nwabor, Ozioma Forstinus \\ Department of Microbiology, University of Nigeria, Nsukka, Nigeria \\ E-mail address: nwaborozed@gmail.com; +2348038781993
}

Keywords: Mosquito, Malaria, Larviciding, Environmental Sanitation, Insecticides

\begin{abstract}
The high prevalence of malaria in Africa has defiled many strategies aimed at its eradication. Researchers from various fields have tried without success in this fight against mosquito and its malaria disease. Annually billions of dollars are spent in the design of programs which are aimed at combating this dreaded disease. However all this spending seems to go down the drain as malaria and its vector mosquitoes celebrate their unflinching victory. Current control measures focusing on ways of preventing the disease vis- a -vis, protect man from the vectors "anopheline mosquito" are the mainstay of malaria prevention and control. Many of these control measures are operational with each contributing in its little way. The use of Long Lasting Insecticide Treated Nets (LLITN) and Indoor Residual Sprays (IRS) are well established strategies with global recognition and currently ongoing in Africa. However, as a result of shortcomings in these major control measures, new strategies with hopes of blissful success are been sought after. Larviciding (abortion of metamorphosis) and constant and adequate environmental sanitation seems to be the next option available for use. This article therefore takes a look at the vector- anopheline mosquito, its ecology, productivity and distribution. It also considers malaria and the various control and preventive measures currently targeted at its eradication.
\end{abstract}

\section{INTRODUCTION}

Mosquitoes are vectors of disease causing agents found within almost all tropical and subtropical countries. Mosquitoes undergo complete metamorphosis, having egg, larval, pupal and adult stages. There are generally six immature stages during mosquito development; the egg stage, four larval stages referred to as 1st-4th instars and the pupal stage. Mosquito larvae are commonly referred to as "wrigglers" and pupae as "tumblers". There are two subfamilies in the mosquito family (Culicidae): Anophelinae (gambiae, funestus, arabiensies) and Culicinae (quinquefasciatus, pipiens, tarsalis, salinarius etc). Most larvae in the subfamily Culicinae hang down just under the water surface by the siphon, whereas anopheline larvae lie horizontally just beneath the water surface supported by small notched organs of the thorax and clusters of float hairs along the abdomen [1]. Anopheline larvae have no prominent siphon. The larvae of An. gambiae breathe atmospheric oxygen through two 'spiracular openings' on the eighth segment of their abdomen and feed by moving brushlike structures on their mouthparts that create a current of water [2]. They filter out microorganisms, particulate organic matter or detritus and biofilm [3, 4]. The larvae undergo four molts (each successively larger), the last of which results in the pupal stage. The pupal stage of mosquitoes does not feed. Pupae give rise to adult mosquitoes in 2 to 4 days. The emergence process begins with splitting of the pupal skin along the back. An emerging adult must dry its wings and groom its head appendages before flying away [5]. Accordingly, this is a critical stage in the survival of mosquitoes. If there is too much wind or wave action, the emerging adult may fall over, becoming trapped on the water surface to die. This is the reason why little if any mosquito breeding occurs in open water, but occurs at the water's edge among weeds. With optimal food and temperature, the time required for development from larva to adult can be as short as 7 days [6]. Adult mosquitoes of both sexes obtain nourishment for basic metabolism and flight by feeding on nectar [5]. In addition, females of most species need a blood meal from birds, mammals, or other vertebrates for egg development. They suck blood via specialized piercing-sucking mouthparts called probosis. 


\section{ECOLOGY OF MOSQUITOES}

Larvae and pupae of mosquitoes are always found in water. Breeding sites may be anything from water in discarded automobile tyres, tins, plastics and the axils of plants, to pools, puddles, swamps, and lakes. It is very important to note that mosquito species differ in their breeding habits, biting behavior, flight range, and so forth. Typical habitats of An. arabiensis and An. gambiae are puddles, shallow ponds, burrow-pits, brick-pits, tyre tracks, ditches, human foot and animal hoof prints which are often created by the activities of humans or domestic animals [7]. These habitats are open, containing no, little or low (grass) aquatic vegetation [8] and are often of a transient nature, as their availability corresponds to precipitation [7]. An. gambiae can colonize a breeding habitat within a few days after the site is created [9]. Besides temporary habitats, An. arabiensis is also found in market garden wells [10] and water storage tanks. A typical characteristic of breeding sites of An. gambiae is their shallow nature. [11] showed that water bodies inhabited by $A n$. arabiensis were on average $18.0(95 \% \mathrm{CI} \pm 3.5) \mathrm{cm}$ deep, by An. gambiae $29.4( \pm 10.7) \mathrm{cm}$ and by both species $9.7( \pm 4.1) \mathrm{cm}$ on the average. In another field study, average depths of $6.2( \pm 5.3 \mathrm{SD})$ and $10.6( \pm 7.2) \mathrm{cm}$ were recorded in dirt tracks and in ditches, respectively [7].

Despite the dogma that An. gambiae is most often found in turbid water collections, various studies that examined the characteristics of larval habitat or larval population dynamics, failed to give a clear relationship between the presence of immatures and the clarity of breeding sites. It is known that dark substrates receive more eggs than light ones and moist substrates more than dry ones [12]. An. gambiae was hence concluded to prefer turbid water over clear water [13]. This was supported by [11] who observed that An. gambiae and An. arabiensis were associated with habitats that were high in turbidity and that both species increased in larval densities with increasing water turbidity. In contrast, [14] found that An. gambiae preferred clear rainwater over natural water from forests and natural wetlands, which contained more impurities and was supported by [15] who showed a preference of An. gambiae to breed in rather clear water bodies. Other factors that may play an important role in habitat selection are volatile compounds that are produced by microbial populations in the breeding site [16], chlorophyll a content in the breeding site [8] or the presence of conspecific larvae or aquatic predators [17]. Some studies reported no effect of turbidity on the occurrence of An. gambiae [18]. However, An. arabiensis and An. gambiae are often found to share larval habitats [20]. A clear difference in requirements for the larval environment of the two species has not been observed, but is subject of discussion. Several studies suggest the requirements are similar [11], others think they differ, but were unable to show that explicitly [14].

\section{HABITAT PRODUCTIVITY}

Mosquito breeding site productivity, estimated in terms of the numbers and size of mosquitoes produces over time depends, not only on the initial number of eggs that are deposited, but on the growth, development rate and survival of the mosquito immatures. Larval developmental rate, survival and adult size affect the transmission of malaria. The time to develop from an egg into an adult, combined with larval survivorship, determines the numbers of emerging mosquitoes over time. The size of the emerging adults is of importance, as larger females have been found to survive longer and have a greater fecundity [20]. Smaller and virgin females on the other hand require a second or third blood meal in order to develop mature eggs, prolonging the time to their first oviposition [21]. Intermediate-sized mosquitoes were found to be more infectious to humans [21]. Besides size, various biotic and abiotic factors also affect the growth, development and survival of the immature mosquitoes and consequently affect habitat productivity [22]. Under laboratory conditions, where larvae were exposed to constant temperatures, [23] showed that larvae took 9.8 to 23.3 days to develop into adults, depending on the temperature. Another laboratory study investigated the duration between oviposition and pupation and reported a time period between 7 and 27 days [24]. In another field study, it was shown that the duration of the immature lifetime of Anopheles gambiae ranges from 8 to 22 days in habitats of different size [25]. Eggs hatch within one day, larvae grow into pupa within 6-19 days and the pupal stage lasts 1-2 days. A similar field 
study by [25] observed a shorter time range of the development from egg to adult, which was 8.411.5 days. [26] observed that larvae, newly hatched from the eggs, took on average 11.8 days to develop into adults, in small ponds and pools, ditches and rice fields. The mortality observed among the immature stages of An. gambiae in the field is extremely high. In all, only a small fraction (2$8 \%$ ) of the larvae eventually survives to the adult stage [27]. It is highly likely that many biotic and abiotic variables, interact and a combination of these factors affect the productivity of a breeding site [10]. In general it is believed that; nutrition, larval densities and water temperature are the principal contributing factors that affect growth and development of mosquito immatures [4].

\section{SPATIAL AND TEMPORAL DISTRIBUTION}

Mosquito species differs in their distribution within the environment. Among the specie Anopheles, An. gambiae is usually the predominant species in wet environments with high humidity whereas An. arabiensis is more common in hotter zones with less rainfall [28]. However, both species occur sympatrically across a wide range of tropical Africa [29]. Breeding of An. gambiae is mostly restricted to the rainy seasons with larval and adult densities increasing rapidly and the species predominating over An. arabiensis, and An. funestus which are more dominant species during the dry periods $[11,30]$. The distance between oviposition site and blood host may affect the oviposition choice [17]. [30] showed that immatures of An. gambiae would be found in breeding sites closer to houses and further away from cowsheds and a study [31] showed that significantly more larvae of An. arabiensis than An. gambiae were collected in pools close to cattle and suggested that species distribution may be explained to a large extent by the presence of suitable hosts instead of breeding site availability.

\section{Gonotrophic cycle}

The gonotrophic period or gonotrophic cycle is defined as the time period between two ovipositions. This period includes the search for a host, the ingestion and digestion of a blood meal, the maturation of the ovaries and the search for a suitable aquatic breeding site to deposit the mature eggs. Each gonotrophic cycle lasts about 2-4 days for An. gambiae [32], but its length will depend on factors such as breeding site availability [33], number of previous gonotrophic cycles and temperature [34, 35]. In the field only a small percentage of females of An. gambiae survive for more than three or four gonotrophic cycles [36]. Although a small percentage was found to survive for over ten cycles [37].

\section{Malaria burden}

Fifty-five percent $(55 \%)$ of the world's population was reported at risk of mosquito borne diseases in 124 countries [38],. In the first comprehensive report on the Roll Back Malaria partnership, malaria was said to be endemic in 117 countries with some 3.2 billion people living in risk areas all over the world [39]. Another report further stated that each year, there are about 350500 million clinical cases of malaria worldwide with over 1 million death. About $59 \%$ of all clinical cases occur in Africa, 38\% in Asia, and 3\% in the Americas [40]. Malaria mortality is also highest in Africa with $89 \%$ of all deaths whereas $10 \%$ occurs in Asia and less than $1 \%$ in the Americas. Of all malaria cases caused by Plasmodium falciparum, the most deadly human malaria species, 74\% are in Africa, $25 \%$ in Asia and $1 \%$ in the Americas. Anopheline mosquitoes are the vectors responsible for the transmission of the deadly malaria etiological agent "plasmodium" [5]. Despite several efforts in the field of vector control, the medical and economic burden caused by vectorborne diseases including malaria continues to grow, plaguing the continent Africa with no visible remedy in sight.

\section{Malaria Prevention and Control}

Despite the huge investment and intensive research in the development of malaria vaccine, science is yet to record a break through. However, a number of effective preventive methods are currently utilized to combat malaria. The policies and prevention strategies used are defined by the 
available resources and epidemiological setting of the diseases [41]. Environmentally, to prevent these diseases, the mosquito population must be kept at a low level at all times. The most effective way to control the mosquito population is to get rid of their breeding sources [42]. As far as possible, stagnant waters should be removed permanently by good and regular housekeeping practices such as filling up ground depressions, disposing discarded containers properly and clearing choked drains and roof gutters. For those mosquito breeding habitats that cannot be removed permanently, a competent pest control operator should be engaged to look out for them within premises and treat them with insecticides to prevent breeding. Prevention of malaria encompasses a variety of measures that may protect against being bitten by the disease vector or against the development of disease in infected individuals [43]. Full coverage and access to prevention methods is the means to reducing malaria incidence and eradicating the disease. There are three primary prevention strategies that are currently being utilized by 107 malarious countries. The first is drug treatment, the second is indoor residual spraying to eradicate mosquitoes, and the third, is mosquito nets to prevent bites [44].

\section{Drug treatment}

Given the increasing incidence of resistance to previous drugs used in malaria therapy, current malaria drug treatment focuses on combination drug therapies as recommend by the World Health Organization. The synergistic effect of these drugs are employed as the resistance of the disease to conventional drug therapies, such as chloroquine, sulfadoxine pyrimethamine (SP) and amodiaquine, has increased. Artemisinin-based Combined Therapies (ACTs) are the most effective drug treatments currently. They produce a very rapid therapeutic response to malaria. Since 2001, 42 malaria-endemic countries have started using ACTs [44, 45]. Unfortunately, there seem to be an impending relapse as resistance of plasmodium to certain Artemisinin based combined therapies emerges.

\section{Indoor residual spraying (IRS)}

IRS is a highly-effective strategy for combating malaria and may provide a lasting impact in areas of intense transmission. Unfortunately, the availability of low-risk and cost-effective insecticides is diminishing due to increasing mosquito resistance and little development of new compounds over the past 20 years. Approximately 50\% of African nations currently use the IRS in malaria control $[44,45]$. However, despite the use of IRS, malaria remains a major Public Health problem in Africa. To date, IRS has only been implemented in Nigeria in a limited fashion. However, according to the National Malaria Strategic Plan 2009-2013, the objective was to gradually scale up spraying to cover $20 \%$ of households nationwide (or almost seven million households) by 2013 [45].

\section{Mosquito nets}

Mosquito nets, particularly insecticide-treated nets, are a highly recommended strategy for the prevention of malaria. Mosquito nets serve as the principal prevention strategy against malaria because they are cost-effective, efficacious, and more available than other strategies. Long-lasting insecticide nets have recently been developed and provide protection for up to five years. Most of the mosquitoes that carry the malaria parasite bite individuals during the night hence bed nets protect individuals from the mosquitoes during this time by preventing contact and thus reducing the risk of malaria. Furthermore, if treated with the insecticide, the net repels mosquitoes and shorten the life of the mosquito [44]. The use of mosquito nets has consistently shown a reduction in malaria cases and overall mortality related to malaria [45]. Twelve insecticides from four classes (organochlorines, organophosphates, carbamates and pyrethroids) have been recommended for IRS $[46,47]$, but only pyrethroids have been approved for treating bed nets. Since the mid-1950s, there have been numerous reports of reduced Anopheles susceptibility to DDT, malathion, fenithrotion, propoxur and bendiocarb, and resistance to all four classes of insecticides has been found in Anopheles species in different parts of Africa [48, 49]. A much more recent development is that of 
pyrethroid resistance with cross-resistance to DDT, first reported in Anopheles gambiae from Côte d'Ivoire [50] and now widespread in West Africa. Pyrethroid-DDT cross-resistance presents a major challenge for malaria vector control in Africa because pyrethroids represent the only class of insecticides approved for treating bed nets and DDT is recommended for use in IRS [51].

\section{Larval control}

Larval control is the foundation of most mosquito control programs. Whereas adult mosquitoes are widespread in the environment, larvae must have water to develop. Control efforts therefore can be focused on aquatic habitats. Minimizing the number of adults that emerge (aborting their development) is crucial to reducing the incidence and risk of disease. The three key components of larval control are environmental management, biological control, and chemical control. Larviciding is a general term for killing immature insects by applying agents, collectively called larvicides, to control larvae and/or pupae stages of these insects [52]. This is an evolving control measure that targets the larva stage of the mosquito. Many people think that the best time to begin a mosquito control program is when the numbers of biting female mosquitoes reach an intolerable level. Contrary to this believe, the best time to begin a mosquito management program is before the adult mosquitoes emerge. Control efforts should begin immediately after the mosquito eggs have hatched, the breeding site should be inspected, and the numbers of larvae present quantified to determine whether or not the use of an insecticide is justified [40]. Mosquitoes are most efficiently and economically destroyed when they are in the larval stage and are concentrated in their breeding site. Preventing the larvae from becoming adult mosquitoes minimizes the area that would have to be treated. It also prevents the development of an annoyance or health problem and it reduces the potential environmental impacts of the adult mosquito control program [40]. Larviciding can reduce overall insecticide use in a mosquito control program by reducing or eliminating the need for ground or aerial application of insecticides to kill adult mosquitoes [41]. [53] considered mosquitoes in the larval stage an attractive target for pesticides because they breed in water and, thus, are easy to deal with in this habitat whereas $[54,55]$ posited that larviciding is a preferred option in vector control because larvae occur in specific areas and can thus be more easily controlled. Treatment of mosquito breeding sites provides control before the biting adults appear and disperse from such sites.

\section{Chemical larvicides}

Chemical larvicides/pesticides are rarely used to control mosquito larvae. Organophosphate larvicides are used infrequently because of their potential non-target effects and label restrictions. Temephos is currently the only organophosphate registered for use as a larvicide in California [56]. This product can be safely and effectively used to treat temporary water or highly polluted water where there are few non-target organisms and/or livestock are not allowed access. The efficacy of temephos may be up to 30 days depending on the formulation [56]. In Nigeria, and most Africa nations chemical larvicides are currently not in existence and are generally unknown and unheard of. Chemical adulticides (insecticides) rather are all that seem to be as the populace unaware of the potential threat posed by the larva burden themselves with the adult insect leaving out the larva which breeds at every nock and cranny of their environment.

\section{Microbial insecticides}

Microbial insecticides are formulated to deliver a natural toxin to the intended target organisms. Bacteria are single-celled parasitic or saprophytic microorganisms that exhibit both plant and animal properties and range from harmless and beneficial to intensely virulent and lethal. Bacillus thuringiensis (Bt), is the most widely used agricultural microbial pesticide in the world, and the majority of microbial pesticides registered with the Environmental Protection Agency (EPA) are based on Bt [52]. The Bt serovar kurstaki (Btk) is the most commonly registered microbial pesticide, and this variety has activity against Lepidoptera (butterflies and moths) larvae [52]. It was originally isolated from natural Lepidopteran die-offs in Germany and Japan. Activity 
of $B t$ against species of mosquitoes were reported [57]. Bt products have been available since the 1950s. In the 1960s and 1970s, the World Health Organization (WHO) encouraged and subsidized scientific discovery and utilization of naturally occurring microbes. As a result of those early studies and a whole body of subsequent work, two lines of mosquito control products have been developed: crystalline toxins of two closely related gram-positive, aerobic bacteria - Bacillus thuringiensis israelensis (Bti) and Bacillus sphaericus $(B s)$. Mosquito control agents based on Bt are the second most widely registered group of microbial pesticides. Highly successful Bti products have expanded the role of microbial agents into the public health arena [56]. However, the use of these microbial insecticides has not received the necessary popularity in tropical and third world countries were the plague of mosquito and its corresponding diseases seem to be concentrated. The use of $B t$ insecticides although proved to be effective also presents a problem given the non selective mode of its action and hence a threat to ecologist, agriculturist, environmentalist and the world at large.

\section{CONCLUSION}

As scientist and researchers continue to battle for a lasting solution that will forever bring to rest this onslaught of malaria, humanity is left with no other option but to protect it's self from the vector mosquito. Prevention and protection from these insects remains the most veritable means to maintaining a malaria free nation or at least reducing its scourge to the barest minimum. However, all of these physical prevention methods require the availability of health infrastructure and education campaigns to effectively implement strategies and educate populations on the need for malaria control. Current malaria vector control, using either insecticide-treated nets (ITNs) or indoor residual spraying (IRS) relies on the continued susceptibility of Anopheles mosquitoes to a limited number of insecticides. Long-lasting insecticidal nets (LLINs) and indoor residual spraying (IRS) are the mainstay of malaria vector control programme because they are highly effective, have a relatively low cost, and their manufacture and distribution can be rapidly scaled up. Unfortunately, with the current trend of resistance to insecticide exhibited by these insect vectors and to toxicity of most of these products which often results in allergy, researchers obviously have to refocus their attention on other strategies which do not rely on the use of insecticides. Other interventions such as environmental management and larviciding can be useful but only under certain conditions, depending on the target vector and the local situation [58]. Vector control is a critical facet of malaria control today and is expected to continue to be so. Vector control remains the single largest category of spending for malaria control by donors. Proper sanitation and environmental management which includes the clearing of bushes within residential areas and destruction of breeding sites such as water logged drainages should be encouraged and scaled up as a means of reducing malaria scourge. Sanitary inspectors should be commissioned and sent to rural neighbourhoods to educate and when necessary prosecute environmental defaulters.

\section{Refrences}

[1] Aymere, A. and Laikemariam, K. (2006). Vector and Rodent Control. Lecture Notes, Degree and Diploma Programs for Environmental Health Science Students. Haramaya University. Pp 17-20.

[2] Merritt, R.W., Dadd, R.H. and Walker, E.D. (1992). Feeding behavior, natural food, and nutritional relationships of larval mosquitoes. Annual Review of Entomology, 37: 349-376.

[3] Clements, A.N. (2000). The Biology of Mosquitoes, Volume 1: Development, Nutrition and Reproduction. New York: CABI publishing. 536 p.

[4] Mutuku, F.M., Alaii, J.A., Bayoh, M.N., Gimnig, J.E., Vulule, J.M., Walker, E.D., Kabiru, E., Hawley, W. A. (2006). Distribution, description, and local knowledge of larval habitats of Anopheles gambiae s.l. in a village in western Kenya. American Journal of Tropical Medicine and Hygiene, 74: 44-53. 
[5] Wendy, C.V., Goddard, J. and Harrison, B. (2012). Identification Guide to Adult Mosquitoes in Mississipp.Mississippi State University Extension Service, 300-04-12.

[6] CDC (2004). Multifocal autochthonous transmission of malaria-Florida, 2003, CDC, MMWR, 53: 412-413.

[7] Koenraadt, C.J.M., Githeko, A.K. and Takken, W. (2004). The effects of rainfall and evapotranspiration on the temporal dynamics of Anopheles gambiae s.s. and Anopheles arabiensis in a Kenyan village. Acta Tropica 90: 141-153.

[8] Mwangangi, J.M., Mbogo, C.M., Muturi, E.J., Nzovu, J.G., Githure, J.I., Yan, G. (2007a). Spatial distribution and habitat characterisation of Anopheles larvae along the Kenyan coast. Journal of Vector Borne Diseases, 44: 44-51.

[9] Minakawa, N., Sonye, G and Yan, G (2005). Relationships between occurrence of Anopheles gambiae s.l. (Diptera: Culicidae) and size and stability of larval habitats. Journal of Medical Entomology, 42: 295-300.

[10] Robert, V., Awono-Ambene, H.P. and Thioulouse, J. (1998). Ecology of larval mosquitoes, with a special reference to Anopheles arabiensis (Diptera: Culicidae) in market-garden wells in urban Dakar, Senegal. Journal of Medical Entomology, 35:948-955.

[11] Gimnig, J.E., Ombok, M., Kamau, L. and Hawley, W.A. (2001). Characteristics of larval anopheline (Diptera: Culicidae) habitats in western Kenya. Journal of Medical Entomology, 38: $282-288$.

[12] Huang, J., Walker, E.D., Vulule, J. and Miller, J.R. (2007). The influence of darkness and visual contrast on oviposition by Anopheles gambiae in moist and dry substrates. Physiological Entomology, 32: 34-40.

[13] Minakawa, N., Mutero, C.M., Githure, J.I., Beier, J.C. and Yan. G. (1999). Spatial distribution and habitat characterization of anopheline mosquito larvae in western Kenya. American Journal of Tropical Medicine and Hygiene, 61: 1010 -1016.

[14] Munga, S., Minakawa, N., Zhou, G., Barrack, O.J and Githeko, A.K, Yan., G. (2005). Oviposition site preference and egg hatchability of Anopheles gambiae: effects of land cover types. Journal of Medical Entomology, 42: 993-997.

[15] Sattler, M.A, Mtasiwa, D., Kiama, M., Premji, Z., Tanner, M., Killeen, G.F., Lengeler, C. (2005). Habitat characterization and spatial distribution of Anopheles sp. mosquito larvae in Dares Salaam (Tanzania) during an extended dry period. Malaria Journal 4(4): 1475-2875.

[16] Sumba, L.A, Guda, T.O, Deng, A.L, Hassanali, A, Beier, J.C, Knols, B.G.J. (2004). Mediation of oviposition site selection in the African malaria mosquito Anopheles gambiae (Diptera: Culicidae) by semiochemicals of microbial origin. International Journal of Tropical Insect Science, 24: 260-265.

[17] Munga, S., Minakawa, N., Zhou, G., Barrack, O.J, Githeko, A.K., Yan, G. (2006). Effects of larval competitors and predators on oviposition site selection of Anopheles gambiae sensu stricto. Journal of Medical Entomology, 43: 221-224.

[18] Mwangangi, J.M., Mbogo, C.M., Muturi, E.J., Nzovu, J.G., Kabiru, E.W., Githure, J.I., Novak, R. J., Beier, J.C. (2007b). Influence of biological and physicochemical characteristics of larval habitats on the body size of Anopheles gambiae mosquitoes (Diptera: Culicidae) along the Kenyan coast. Journal of Vector Borne Diseases, 44: 122127.

[19] Edillo, F.E., Touré, Y.T., Lanzaro, G.C., Dolo, G. and Taylor, C.E. (2002). Spatial and habitat distribution of Anopheles gambiae and Anopheles arabiensis (Diptera: Culicidae) in Banambani village, Mali. Journal of Medical Entomology, 39: 70-77. 
[20] Ameneshewa, B., Service, M.W. (1996). The relationship between female body size and survival rate of the malaria vector Anopheles arabiensis in Ethiopia. Medical and Veterinary Entomology, 10: 170-172.

[21] Lyimo, E.O and Takken, W. (1993). Effects of adult body size on fecundity and the pregravid rate of Anopheles gambiae females in Tanzania. Medical and Veterinary Entomology, 7: 328-332.

[22] Paaijmans, K.P. (2008). Weather, water and malaria mosquito larvae-The impact of meteorological factors on water temperature and larvae of the Afro-tropical malaria vector Anopheles gambiae Giles ISBN 978-90-8504-750-6

[23] Bayoh, M.N. and Lindsay, S.W (2003). Effect of temperature on the development of the aquatic stages of Anopheles gambiae sensu stricto (Diptera: Culicidae). Bulletin of Entomological Research, 93: 375-381.

[24] Chen, H., Fillinger, U. and Yan, G (2006). Oviposition behavior of female Anopheles gambiae in western Kenya inferred from microsatellite markers. American Journal of Tropical Medicine and Hygiene, 75: 246-250.

[25] Gimnig, J.E., Ombok, M., Otieno, S., Kaufman, M.G., Vulule, J.M., Walker, E.D. (2002). Density dependent development of Anopheles gambiae (Diptera:Culicidae) larvae in artificial habitats. Journal of Medical Entomology 39: 162 - 172.

[26] Service, M.W. (1977). Mortalities of the immature stages of species B of the Anopheles gambiae complex in Kenya: comparison between rice fields and temporary pools, identification of predators, and effects of insecticidal spraying. Journal of Medical Entomology, 13: 535-545.

[27] Okogun, G.R.A. (2005). Life-table analysis of Anopheles malaria vectors: generational mortality as tool in mosquito vector abundance and control studies. Journal of Vector Borne Diseases, 42: 45-53.

[28] Kirby, M.J. and Lindsay, S.W. (2004). Responses of adult mosquitoes of two sibling species, Anopheles arabiensis and A. gambiae s.s. (Diptera: Culicidae), to high temperatures. Bulletin of Entomological Research, 94: 441-448.

[29] Coetzee, M., Craig, M. and Le-Sueur, D. (2000). Distribution of African malaria mosquitoes belonging to the Anopheles gambiae complex. Parasitology Today, 16: 74-77.

[30] Minakawa, N., Seda, P. and Yan, G (2002). Influence of host and larval habitat distribution on the abundance of African malaria vectors in western Kenya. American Journal of Tropical Medicine and Hygiene, 67: 32-38.

[31] Charlwood, J.D., and Edoh, D. (1996). Polymerase chain reaction used to describe larval habitat use by Anopheles gambiae complex (Diptera: Culicidae) in the environs of Ifakara, Tanzania. Journal of Medical Entomology, 33: 202-204.

[32] Touré, Y.T., Dolo, G., Petrarca, V., Traoré, S.F., Bouaré, M, Dao, A., Carnahan, J., Taylor, C.E. (1998). Mark -release recapture experiments with Anopheles gambiae s.l. in Banambani village, Mali, to determine population size and structure. Medical and Veterinary Entomology, 12: 74-83.

[33] Gu, W., Regens, J.L., Beier, J.C. and Novak, R.J. (2006). Source reduction of mosquito larval habitats has unexpected consequences on malaria transmission. Proceedings of the National Academy of Sciences, 103: 17560-17563. 
[34] Afrane, Y.A., Lawson, B.W., Githeko, A.K., Yan, G. (2005). Effects of microclimatic changes caused by land use and land cover on duration of gonotrophic cycles of Anopheles gambiae (Diptera: Culicidae) in western Kenya highlands. Journal of Medical Entomology, 42: 974-980.

[35] Maharaj, R. (2003). Life table characteristics of Anopheles arabiensis (Diptera: Culicidae) under simulated seasonal conditions. Journal of Medical Entomology, 40: 737-742.

[36] Charlwood, J.D., Vij, R. and Billingsley, P.F. (2000). Dry season refugia of malariatransmitting mosquitoes in a dry savannah zone of East Africa. American Journal of TropicalMedicine and Hygiene, 62: 726-732.

[37] Gillies, M.T. and Wilkes, T.J. (1965). A study on the age-composition of populations of Anopheles gambiae Giles and A. funestus Giles in north-eastern Tanzania. Bulletin of Entomological Research, 56: 237-262.

[38] Beatty, M.E., Letson, W., Edgil, D.M., Margolis, H. (2007). Estimating the total world population at risk for locally acquired dengue infection. Proceedings of 56th Annual Meeting of American Society of Tropical Medicine and Hygiene, Philadelphia, Pennsylvania, USA, 4-8.

[39] WHO/UNICEF, (2005). World Malaria Report. Roll Back Malaria partnership, WHO/UNICEF. http://rbm.who.int/wmr2005/.

[40] Centers for Disease Control (CDC) (2007) Malaria facts. http://www.cdc.gov/malaria/facts.htm

[41] Nejla, B.S. (2007). The Possession Versus Use of Mosquito Nets for Children Under Five in Kenya. Faculty of Arts \& Sciences, Georgetown University, Washington DC.

[42] National Environment Agency, (1995). Scope of works for mosquito control. Environmental health department, USA.

[43] WHO, (1992). Vector resistance to pesticides. 15th Report of the Export Committee on Vector Biology and Control. WHO. Technical. Report. Series. 818.

[44] WHO, (2005). Guidelines for Laboratory and Field Testing of Mosquito Larvicides. WHO communicable disease control, prevention and eradication. WHO pesticide evaluation scheme. WHO/CDS/WHOPES/GCDPP/2005.13.

[45] Roll Back Malaria, (2006). Country Needs Assessment. http://www.rbm.who.int/cmc_upload/0/000/015/362/RBMInfosheet_11.htm.

[46] Kelly-Hope, L., Ranson, H. and Hemingway, J. (2008): Lessons from the past: managing insecticide resistance in malaria control and eradication programmes. Lancet Infectious Diseases, 8:387-389.

[47] Najera, J.A. and Zaim, M (2002). Decision making criteria and procedures for the judicious use of insecticides. In vol.WHO/CDS/WHOPES/.5 Rev.1. Geneva: World Health Organization.

[48] Awolola, T.S., Brooke, B.D., Hunt, R.H. and Coetze, M. (2002). Resistance of the malaria vector Anopheles gambiae s.s. to pyrethroid insecticides, in south-western, Nigeria. Annals of Tropical Medical Parasitology, 96:849-852

[49] N'Guessan, R., Darriet, F., Guillet, P., Carnevale, P., Traore-Lamizana, M., Corbel, V., Koffi, A. A. and Chandre, F (2003). Resistance to carbosulfan in Anopheles gambiae from Ivory Coast, based on reduced sensitivity of acetylcholinesterase. Medical and Veterinary Entomology, 17:19-25 
[50] Elissa, N., Mouchet, J., Riviere, F., Meunier, J. Y. and Yao, K, (1993). Resistance of Anopheles gambiae s.s. to pyrethroids in Cote d'Ivoire. Ann soc Belg Med Tropica, 73: 291294

[51] WHO, (2006). Pesticides and their Application for the Control of Vectors and Pests of Public Health Importance. Geneva, WHO Pesticide Evaluation Scheme

[52] Florida Mosquito Control (2009). Florida Coordinating Council on Mosquito Control: The state of the mission as defined by mosquito controllers, regulators, and environmental managers. Vero Beach, FL: University of Florida, Institute of Food and Agricultural Sciences, Florida Medical Entomology Laboratory

[53] Nandita, C., Anupam, G. and Goutam, C. (2008). Mosquito larvicidal activities of Solanum villosum berry extract against the dengue vector Stegomyia aegypti. BMC Complementary \& Alternative Medicine, 1186/1472-6882-8-10

[54] Ubulom, M. E., Imandeh, N. G., Udobi, C.E. and Ilya, I. (2012). Larvicidal and Antifungal Properties of Picralima nitida (Apocynaceae) Leaf Extracts. European Journal of Medicinal Plants, 2(2): 132-139

[55] Nwabor, Ozioma F., Dibua, Esther U. M , Nnamonu, Ikechukwu E., Odiachi, Osita, Edeh, Gloria O, Ezechukwu, Samuel C, Rex E. T., Stanislaus I. (2014). An investigation of the lethality of Picralima nitida, Family Apocynaceae in malaria vector control. Advances in Life Science and Technology; 23:77-82.

[56] CDPH, (2010). Best Management Practices for Mosquito Control on California State Properties: http://www.westnile.ca.gov/resources.php

[57] Omoya, F.O. and Akinyosoye, F.A. (2011). Evaluation of larvicidal potency of some entomopathogenic bacteria isolated from insect cadavars on Anopheles arabiensis larvae in Nigeria. Int J Pharm Biomed Res., 2(3): 145-148

[58] WHO, (2012). Global Plan for Insecticide Resistance Management in Malaria vector. Geneva. 